\title{
Qubit architecture with high coherence and fast tunable coupling
}

\author{
Yu Chen ${ }^{1},{ }^{*}$ C. Neill ${ }^{1},{ }^{*}$ P. Roushan ${ }^{1},{ }^{*}$ N. Leung ${ }^{1}$, M. Fang ${ }^{1}$, R. Barends ${ }^{1}$, J. Kelly ${ }^{1}$, B. Campbell ${ }^{1}$, Z. Chen ${ }^{1}$, B. \\ Chiaro $^{1}$, A. Dunsworth ${ }^{1}$, E. Jeffrey ${ }^{1}$, A. Megrant ${ }^{1}$, J. Y. Mutus ${ }^{1}$, P. J. J. O’Malley ${ }^{1}$, C. M. Quintana ${ }^{1}$, D. Sank ${ }^{1}$, \\ A. Vainsencher ${ }^{1}$, J. Wenner ${ }^{1}$, T. C. White ${ }^{1}$, Michael R. Geller ${ }^{2}$, A. N. Cleland ${ }^{1}$, and John M. Martinis ${ }^{1 \dagger}$ \\ ${ }^{1}$ Department of Physics, University of California, \\ Santa Barbara, California 93106-9530, USA and \\ ${ }^{2}$ Department of Physics and Astronomy, University of Georgia, Athens, Georgia 30602, USA
}

\begin{abstract}
We introduce a superconducting qubit architecture that combines high-coherence qubits and tunable qubit-qubit coupling. With the ability to set the coupling to zero, we demonstrate that this architecture is protected from the frequency crowding problems that arise from fixed coupling. More importantly, the coupling can be tuned dynamically with nanosecond resolution, making this architecture a versatile platform with applications ranging from quantum logic gates to quantum simulation. We illustrate the advantages of dynamic coupling by implementing a novel adiabatic controlled-Z gate, at a speed approaching that of single-qubit gates. Integrating coherence and scalable control, our "gmon" architecture is a promising path towards large-scale quantum computation and simulation.
\end{abstract}

The fundamental challenge for quantum computation and simulation is to construct a large-scale network of highly connected coherent qubits $[1,2]$. Superconducting qubits use macroscopic circuits to process quantum information and are a promising candidate towards this end [3]. Over the last several years, materials research and circuit optimization have led to significant progress in qubit coherence [4-6]. Superconducting qubits can now perform hundreds of operations within their coherence times, allowing for research into complex algorithms such as error correction $[7,8]$.

It is desirable to combine these high-coherence qubits with tunable inter-qubit coupling; the resulting architecture would allow for both coherent local operations and dynamically varying qubit interactions. For quantum simulation, this would provide a unique opportunity to investigate dynamic processes in non-equilibrium condensed matter phenomena [9-13]. For quantum computation, such an architecture would provide isolation for single-qubit gates while at the same time enabling fast two-qubit gates that minimize errors from decoherence. Despite previous successful demonstrations of tunable coupling [14-23], these applications have yet to be realized due to the challenge of incorporating tunable coupling with high coherence devices.

Here, we introduce a planar qubit architecture that combines high coherence with tunable inter-qubit coupling $g$. This "gmon" device is based on the Xmon transmon design [5], but now gives nanosecond control of the coupling strength with a measured on/off coupling ratio exceeding 1000. We find that our device retains the high coherence inherent in the Xmon design, with the coupler providing unique advantages in constructing single- and two-qubit quantum logic gates. With the coupling turned off, we demonstrate that our architecture is protected from the frequency crowding problems that arise from fixed coupling. Our single-qubit gate fidelity is nearly independent of the qubit-qubit detuning, even when op- erating the qubits on resonance. By dynamically tuning the coupling, we implement a novel adiabatic controlled$\mathrm{Z}$ gate at a speed approaching that of single-qubit gates.

A two-qubit unit cell with tunable coupling is shown in Fig. 1(a). The qubits and control lines are defined

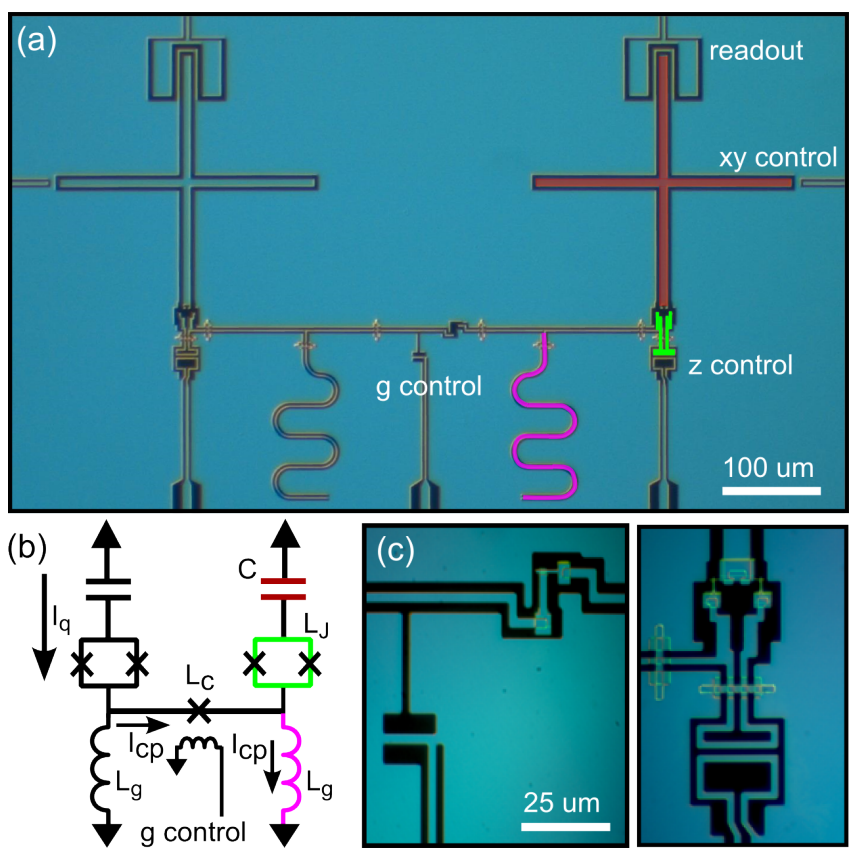

FIG. 1: (a) Optical micrograph of two inductively coupled gmon qubits. The cross-shaped capacitors are placed in series with a tunable Josephson junction and followed by a linear inductor to ground. The circuit is depicted schematically in (b) with arrows indicating the flow of current for an excitation in the left qubit. The qubits are connected with a line containing a junction that acts as a tunable inductor to control the coupling strength. (c) Micrographs of the coupler junction (left) and qubit SQUID (right). The bottom of each image shows a bias line used to adjust the coupling strength (left) and qubit frequency (right, not shown in schematic). 

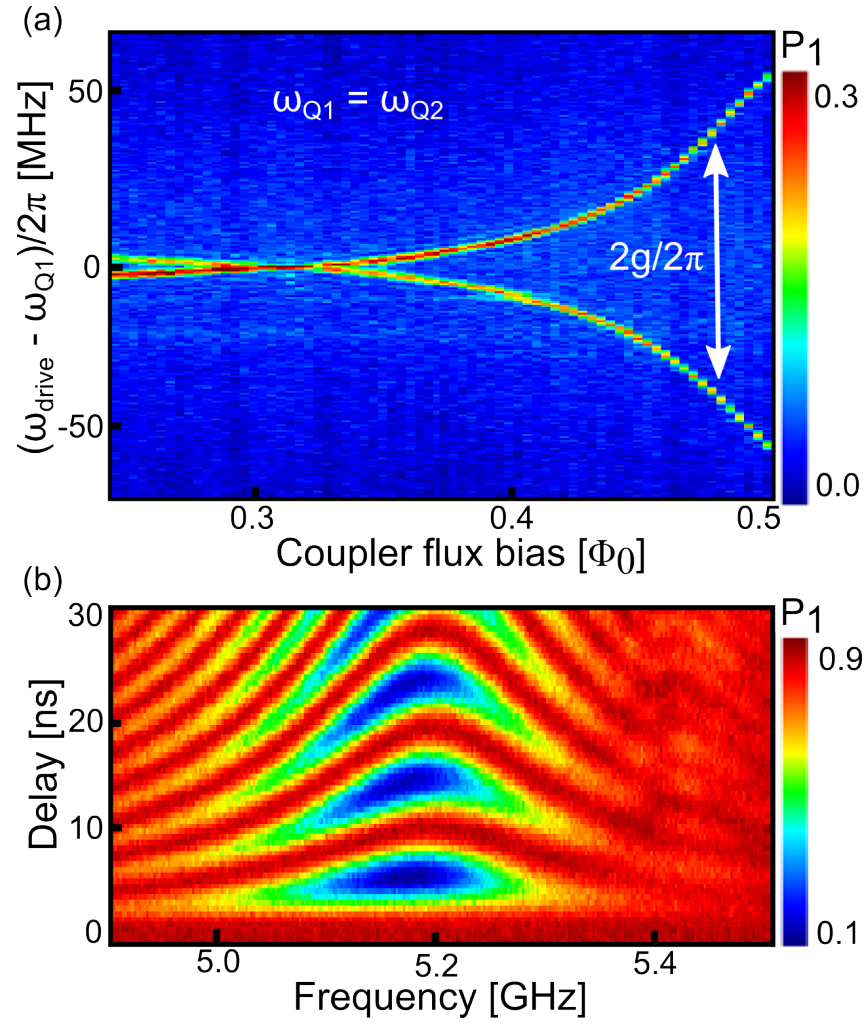

FIG. 2: (a) The dependence of the coupling strength on the coupler flux bias while the two qubits are on resonance, with $\omega_{Q 1} / 2 \pi=\omega_{Q 2} / 2 \pi=5.67 \mathrm{GHz}$. For each value of the coupler flux bias, we sweep the microwave drive frequency and measure the excited state probability $P_{1}$ (colorbar) of $Q_{1}$. There are two distinct peaks in the spectroscopy that result from an energy level splitting. The frequency splitting is twice the coupling strength $g / 2 \pi$ and ranges from 0 to $110 \mathrm{MHz}$. (b) $Q_{1}$ excited state probability (colorbar) versus the frequency of $Q_{1}$ (horizontal axis) after exciting the qubit and waiting a variable delay time (vertical axis). $Q_{2}$ is fixed at $5.18 \mathrm{GHz}$ and the coupling is set to $55 \mathrm{MHz}$. On resonance, the two qubits swap an excitation in $5 \mathrm{~ns}$.

by an aluminum film with cuts exposing the underlying sapphire substrate. Our circuit design is based on the Xmon qubit, consisting of a cross-shaped capacitor resonating with a nonlinear inductor $L_{J}=9.0 \mathrm{nH}$ made from a SQUID. We modify the Xmon design to introduce a linear inductor $L_{g}=200 \mathrm{pH}$ from the junction to ground, with $L_{g} \ll L_{J}$ so that the qubit nonlinearity is largely unaffected (see Ref. [24] for a detailed discussion). This inductor introduces a node in the circuit where current from one qubit can be tapped off to interact with a neighboring qubit. A junction connecting the two nodes acts as a tunable inductance $L_{c}$ that controls the flow of this current and therefore the coupling.

The physics behind this tunable coupler is well explained using a simple linear model, since the coupling currents are much smaller than the critical current of the coupling junction $I_{0}=330 \mathrm{nA}$; see Ref. [25] for a full quantum mechanical treatment. A circuit diagram for the device is given in Fig. 1(b). An excitation current in the first qubit $I_{q}$ mostly flows through $L_{g}$, with a small fraction $I_{\mathrm{cp}}=I_{q} L_{g} /\left(2 L_{g}+L_{c}\right)$ flowing through the coupler to the second qubit. This current generates a flux in the second qubit $\Phi_{2}=L_{g} I_{\mathrm{cp}}$. In the absence of parasitic inductance, the effective mutual inductance can be expressed as

$$
M=\frac{\Phi_{2}}{I_{q}}=\frac{L_{g}^{2}}{2 L_{g}+L_{c}} .
$$

Using this mutual inductance, the interaction Hamiltonian for the two qubits on resonance can be written as

$$
\hat{H}_{\text {int }}=-\frac{\omega_{0}}{2} \frac{M}{L_{J}+L_{g}}\left(\hat{a}_{1}^{\dagger} \hat{a}_{2}+\hat{a}_{1} \hat{a}_{2}^{\dagger}\right)
$$

where $\omega_{0}$ is the qubit resonance frequency. This equation uses the rotating wave approximation to express photon swapping via the raising and lowering operators [23]. The coefficient of the interaction Hamiltonian gives the coupling strength

$$
g=-\frac{\omega_{0}}{2} \frac{L_{g}}{L_{J}+L_{g}} \frac{L_{g}}{2 L_{g}+L_{c 0} / \cos \delta},
$$

where $L_{c}$ is replaced by the Josephson inductance $L_{c}=$ $\Phi_{0} /\left(2 \pi I_{0} \cos \delta\right) \equiv L_{c 0} / \cos \delta$. Here $\delta$ is the phase difference across the coupler junction, set by applying a DC flux. Note that the DC current from this flux flows only through the coupler, not through the qubit junctions because of their series capacitance. The coupling $g$ can be varied continuously from negative to positive, going smoothly through zero at $\delta=\pi / 2$. This smooth transition from positive to negative ensures the existence of a bias such that the coupling is completely negated; this is true even in the presence of small stray coupling.

A critical part of our design is the compatibility between high coherence and tunable coupling. The key concept in maintaining coherence is the voltage divider created by $L_{J}$ and $L_{g}$ : placing the coupling circuit at this low voltage node reduces capacitive losses by a factor of $\left(L_{J} / L_{g}\right)^{2}$ - over 2000 in our design. For the gmon, we measure an energy relaxation time $T_{1} \sim 7-10 \mu \mathrm{s}$ and is independent of the coupling strength (see Ref. [24]). This is comparable to the performance of previous Xmon devices with similar capacitor geometry $(8 \mu \mathrm{m}$ center trace, $4 \mu \mathrm{m}$ gap) and aluminum deposition conditions (high vacuum e-beam evaporation). Devices grown with molecular beam epitaxy and with optimized capacitor geometry have been shown to have lifetimes exceeding $40 \mu \mathrm{s}$ [5].

The core functionality of the gmon coupler is demonstrated in Fig. 2. In panel (a) we show the variation of the coupling strength as a function of the coupler flux bias, for the condition where the two qubits are brought into resonance at a frequency $\omega_{0} / 2 \pi=5.67 \mathrm{GHz}$. Here for one 


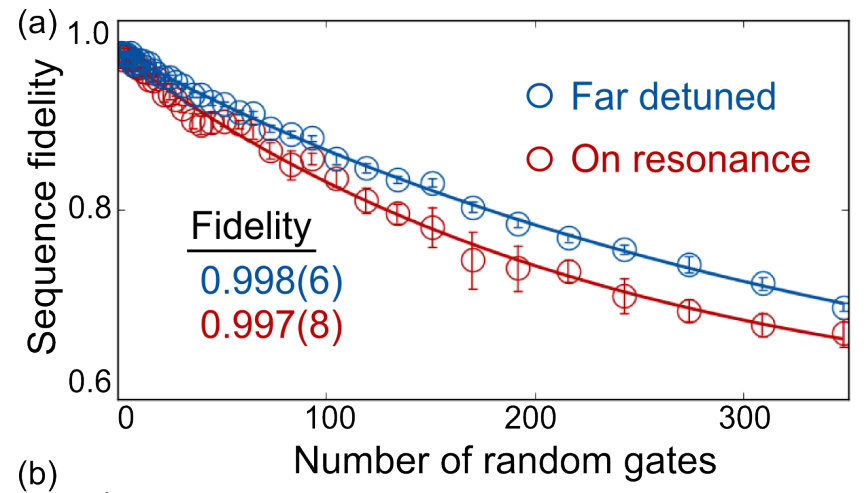

(b)

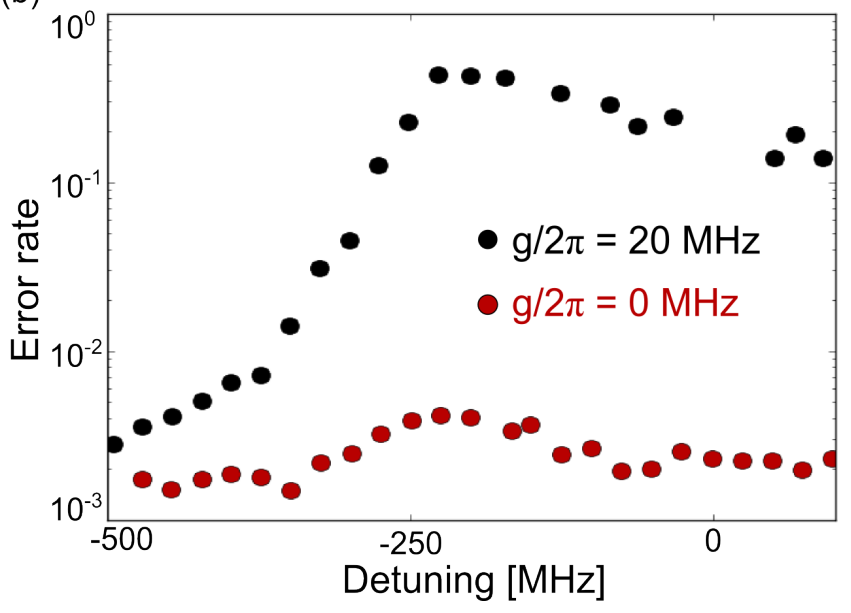

FIG. 3: Simultaneous single-qubit randomized benchmarking. (a) The raw benchmarking data for $Q_{1}$ when $Q_{2}$ is far detuned (blue) and on resonance with random gates applied to both qubits (red). Operating the qubits on resonance degrades the gate performance by $<.1 \%$. Lines are fits to a decaying exponential. (b) The average error rate for $Q_{1}$ as a function of the detuning between the two qubits, shown in red for nominally zero coupling and in black for $20 \mathrm{MHz}$ coupling. The ability to turn off the coupling results in an error rate that is nearly flat, with a value on resonance that is two orders of magnitude lower than for moderate fixed coupling.

qubit we sweep the microwave drive frequency and measure the qubit excited state probability $P_{1}$. We observe two distinct resonances at frequencies $\omega_{0}+g$ and $\omega_{0}-g$ that result from the coupling-induced energy level splitting. The total splitting is twice the coupling strength, ranging from 0 to $110 \mathrm{MHz}$. This range can be further increased by modifying the critical current of the coupler junction. Note that we have compensated for the small changes in the qubit frequency $(\sim g)$ that occur as $L_{c}$ is varied;Ref. [24] gives details on how these controls are effectively made orthogonal.

For the data in Fig. 2(b) we set the the coupling strength to its maximum value and rapidly exchange an excitation between the two qubits. We excite the first qubit $\left(Q_{1}\right)$, turn on the coupling, wait a variable delay time, and then measure the excited state probability of $Q_{1}$. We vary the frequency of $Q_{1}$ while fixing that of the second qubit $\left(Q_{2}\right)$. The resonance interaction results in the expected chevron pattern [27]. The strong coupling allows the excitation to swap between the two qubits in $5 \mathrm{~ns}$, consistent with the $110 \mathrm{MHz}$ splitting measured above. At this rate, a $\sqrt{i \text { SWAP }}$ gate could generate a Bell state in $2.5 \mathrm{~ns}$, whereas a non-adiabatic CZ could be implemented in $10 \mathrm{~ns}$ [28]. We have also performed the same measurement with nominally zero coupling (seeRef. [24]) and observe no indication of swapping after $6 \mu \mathrm{s}$. This places an upper bound on the residual coupling of $50 \mathrm{kHz}$, providing an on/off ratio of over 1000 .

By incorporating tunable coupling with high coherence qubits, our architecture provides a viable platform for both quantum computation and simulation. We have applied this device to quantum simulation in a separate experiment where we have demonstrated an interactiondriven topological phase transition [29]. In the following, we focus on applications in quantum computation by implementing elementary logic gates. This architecture offers two distinct advantages: the ability to decouple qubits for local single-qubit gates and the ability to dynamically tune the interaction for fast two-qubit gates.

We characterize gate performance using a simplified form of randomized benchmarking [30, 31], which applies a series of Pauli gates that move the qubit among the 6 states $\{|0\rangle,|1\rangle,(|0\rangle+|1\rangle) / \sqrt{2},(|0\rangle+i|1\rangle) / \sqrt{2},(|0\rangle-$ $|1\rangle) / \sqrt{2},(|0\rangle-i|1\rangle) / \sqrt{2}\}$. These gates belong to a subset of the Clifford group and are generated using microwave pulses that correspond to Bloch sphere rotations of angle $\pi$ and $\pi / 2$ around the $\mathrm{X}$ and $\mathrm{Y}$ axis. From this set we randomly choose $m$ gates and apply these to the qubit, including a final gate that ideally maps the qubit back into the ground state. The probability of finding the qubit in the ground state is called the sequence fidelity $F_{\text {seq }}$, which decays exponentially with the number of gates by $F_{\text {seq }}=A p^{m}+B$. Here $A, B$ and $p$ are fit parameters; $A$ and $B$ relate to state preparation and measurement. We are interested in the average error per gate $r$, determined through the relation $r=(1-p)(d-1) / d$ where $d=2^{N_{\text {qubits }}}$. We note that Pauli gates do not fully depolarize errors, hence the extracted gate fidelities are only indicative.

The ability to isolate individual qubits for local operations is one advantage offered by a tunable coupling architecture. A metric to quantify this isolation is single-qubit gate fidelity $1-r$. As a baseline, we perform randomized benchmarking on the first qubit while the second qubit is far detuned and effectively decoupled. The sequence fidelity is plotted in Fig. 3(a) and displays the expected exponential decay with the number of random gates. Fitting the decay curve yields an average single-qubit gate fidelity of $99.86 \%$. The two qubits are then placed on resonance with $g=0$ and the measurement is repeated on both qubits; data for the first qubit is shown. Simultaneously operating the two qubits on resonance reduces the gate fidelity by $<0.1 \%$. The added error results from 


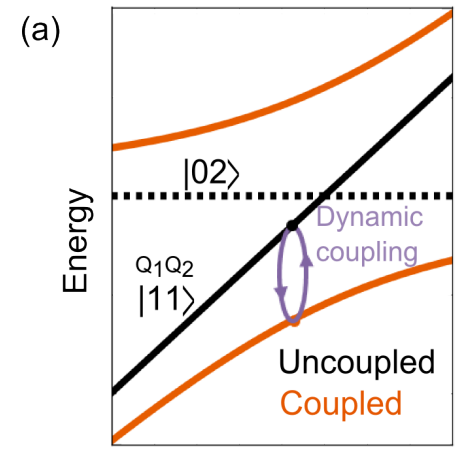

(c)

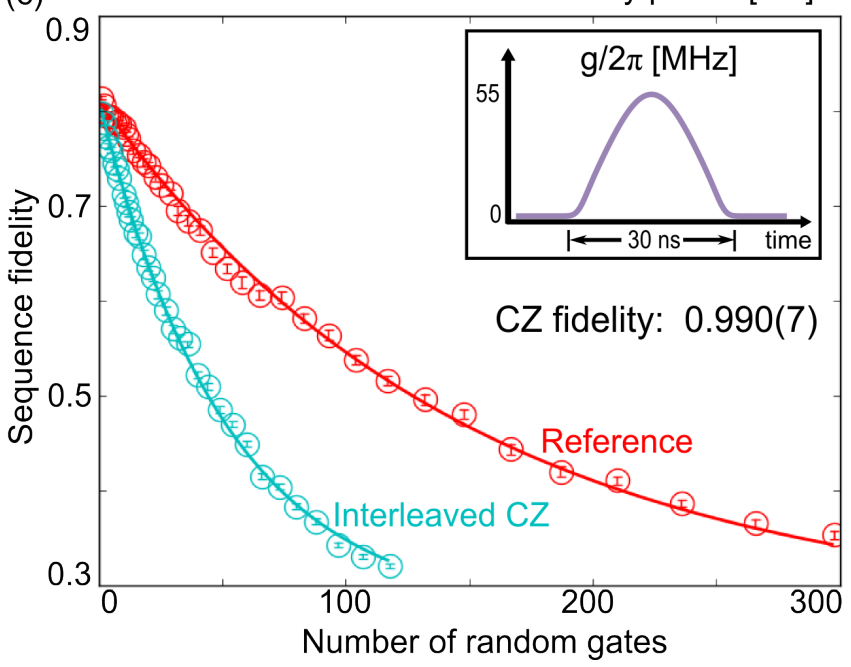

FIG. 4: (a) Energy level diagram; illustration of a CZ using tunable coupling. Black lines are the uncoupled two-photon eigenenergies; orange lines are the coupled eigenenergies. As the coupling is tuned on and off (depicted in purple), the energy levels repel and the states accumulate a dynamic phase. (b) Ramsey data demonstrating zero phase shift for singlephoton states and a $\pi$ phase shift for the two-photon state. (c) Randomized benchmarking results for a $\mathrm{CZ}$ gate utilizing the pulse shape shown inset. We are able to achieve $99.07 \%$ fidelity with a 30 ns gate.

two sources: residual inter-qubit coupling and imperfect cancellation of microwave crosstalk between the control signals.

In panel (b), we repeat this measurement as a function of the frequency separation of the two qubits, demonstrating the effects of frequency crowding that result from fixed coupling. The average error rate is plotted in Fig. 3(b) for both $g / 2 \pi=0$ and $20 \mathrm{MHz}$; note that the latter value is only a third of the maximum possible coupling. Even for this relatively weak interaction, the single-qubit gate fidelity undergoes a significant reduction for detuning less than $500 \mathrm{MHz}$. The ability to turn off the coupling $g$ results in an error rate that is nearly flat, with a value on resonance that is two orders of magnitude lower than for fixed coupling. We note there is a slight degradation in the qubit performance near the qubit nonlinearity (220 MHz).

A concern in designing transmon has been the cross coupling of qubits. One approach to resolve this has been the use of $3 \mathrm{D}$ devices in which qubits are shielded in enclosed boxes [6]. Here we have directly demonstrated that the effects of cross-coupling on fidelity can be made small for planar integrated circuits.

Control over the interaction strength with nanosecond resolution provides a unique tool for constructing fast two-qubit gates. In Fig. 4(a) we illustrate a method for using dynamic coupling to implement a fast controlled-Z (CZ) gate, which has minimal non-adiabatic leakage errors. The straight lines correspond to the energies of the $|11\rangle$ and $|02\rangle$ states of the uncoupled system. Turning on the interaction pushes the energy levels apart, with the energies of the coupled system plotted as curved lines. Adiabatically turning on and off the coupling, as depicted with arrows, causes the $|11\rangle$ eigenstate to accumulate a dynamic phase. By calibrating the length of the interaction the phase shift can be set to $\pi$ for a CZ gate.

In Fig. 4(b) we use a Ramsey measurement to verify that the gate sequence produces the desired results. We first apply a $\pi / 2$ pulse to $Q_{1}$, perform a CZ, apply a second $\pi / 2$ pulse with varying phase, and then measure the qubit excited state probability. We then repeat the experiment with an excitation in $Q_{2}$ and overlay the data. The solid lines are fits to cosine oscillations with zero and $\pi$ phase shifts. The $\pi$ phase shift is observed only when there is an excitation in each qubit, otherwise the phase accumulation is zero.

We extract the fidelity of this $\mathrm{CZ}$ gate using interleaved randomized benchmarking, in which we insert a $\mathrm{CZ}$ between random single-qubit Pauli gates. A reference curve without the interleaved $\mathrm{CZ}$ is measured and plotted in Fig.4(c) along with the interleaved sequence fidelity. Fitting these two curves allows us to extract an average CZ gate fidelity of $99.07 \%$. The dominant error $(\sim 0.66 \%)$ comes from decoherence, measured by interleaved randomized benchmarking on the two qubit idle gate (seeRef. [24]). This error can be suppressed by incorporating optimized capacitor geometry and improved film growth conditions. Surprisingly, despite the short gate time, the non-adiabatic error resulting from leakage to the $|02\rangle$ state is small $(\sim 0.25 \%)$, measured by the Ramsey error filter technique (seeRef. [24]) [32]. This is the result of an optimized adiabatic trajectory based on a theory of optimal window functions [33]. The adiabatic trajectory used to vary the coupling strength is shown in the inset in panel (c).

High-fidelity gates have been demonstrated using Xmon qubits [7]. We believe that these fidelities can be further improved by utilizing the added functionality provided by tunable coupling. This will require the incorporation of lower loss materials, optimized capacitor geometry and characterization using the full Clifford group; this is currently in progress. 
In conclusion, we have demonstrated a superconducting qubit architecture with high coherence and tunable coupling. We explore two distinct advantages of this architecture for quantum computation. First, the ability to isolate individual qubits allows for high fidelity local operations that are not degraded by the presence of neighboring qubits. Second, by dynamically tuning the interaction strength, we demonstrate a new two-qubit CZ gate, at a speed approaching that of single-qubit gates. Combining these features with high coherence, the introduced architecture represents a viable platform for implementing future quantum algorithms.

This work was supported by the Office of the Director of National Intelligence (ODNI), Intelligence Advanced Research Projects Activity (IARPA), through the Army Research Office grant W911NF-10-1-0334. C.N., P.R. and M.R.G acknowledge the support form the US National Science Foundation under CDI grant DMR1029764. All statements of fact, opinion or conclusions contained herein are those of the authors and should not be construed as representing the official views or policies of IARPA, the ODNI, or the U.S. Government. Devices were made at the UC Santa Barbara Nanofabrication Facility, a part of the NSF-funded National Nanotechnology Infrastructure Network, and at the NanoStructures Cleanroom Facility

* These authors contributed equally to this work

$\dagger$ Electronic address: martinis@physics.ucsb.edu

[1] M. A. Nielsen and I. L. Chuang, Quantum Computation and Quantum Information (Cambridge University Press, 2000).

[2] J. Q. You and F. Nori, Physics Today 58, 42 (2005).

[3] M. H. Devoret and R. J. Schoelkopf, Science 339, 1169 (2013).

[4] A. Megrant et al., Appl. Phys. Lett. 100, (2012).

[5] R. Barends et al., Phys. Rev. Lett. 111, 080502 (2013).

[6] H. Paik et al., Phys. Rev. Lett. 107, 240501 (2011).

[7] R. Barends et al., arXiv:1402.4848 (2014).

[8] J. M. Chow et al., arXiv: 1311.6330 (2013).
[9] I. Buluta and F. Nori, Science 326, 108 (2009).

[10] A. A. Houck, H. E. Tureci, and J. Koch, Nat Phys 8, 292 (2012).

[11] Michael. R. Geller et al., arXiv:1210.5260 (2012).

[12] A. Polkovnikov, K. Sengupta, A. Silva, and M. Vengalattore, Rev. Mod. Phys. 83, 863 (2011).

[13] P. Calabrese, F. H. L. Essler, and M. Fagotti, Journal of Statistical Mechanics: Theory and Experiment 2012, P07016 (2012).

[14] M. S. Allman, F. Altomare, J. D. Whittaker, K. Cicak, D. Li, A. Sirois, J. Strong, J. D. Teufel, and R. W. Simmonds, Phys. Rev. Lett. 104, 177004 (2010).

[15] R. C. Bialczak et al., Phys. Rev. Lett. 106, 060501 (2011).

[16] R. Harris et al., Phys. Rev. Lett. 98, 177001 (2007).

[17] T. Hime, P. A. Reichardt, B. L. T. Plourde, T. L. Robertson, C.-E. Wu, A. V. Ustinov, and J. Clarke, Science 314, 1427 (2006).

[18] A. O. Niskanen, K. Harrabi, F. Yoshihara, Y. Nakamura, S. Lloyd, and J. S. Tsai, Science 316, 723 (2007).

[19] S. H. W. van der Ploeg, A. Izmalkov, A. M. van den Brink, U. Hubner, M. Grajcar, E. Il'ichev, H.-G. Meyer, and A. M. Zagoskin, Phys. Rev. Lett. 98, 057004 (2007).

[20] S. J. Srinivasan, A. J. Hoffman, J. M. Gambetta, and A. A. Houck, Phys. Rev. Lett. 106, 083601 (2011).

[21] A. Blais, A. M. van den Brink, and A. M. Zagoskin, Phys. Rev. Lett. 90, 127901 (2003).

[22] Y.-x. Liu, L. F. Wei, J. S. Tsai, and F. Nori, Phys. Rev. Lett. 96, 067003 (2006).

[23] R. A. Pinto, A. N. Korotkov, M. R. Geller, V. S. Shumeiko, and J. M. Martinis, Phys. Rev. B 82, 104522 (2010).

[24] See supplemental materials.

[25] Michael R. Geller, E. Donate, Yu Chen, C. Neill, P. Roushan, John. M. Martinis In preparation (2014).

[26] M. Neeley et al., Phys. Rev. B 77, 180508 (2008).

[27] M. Hofheinz et al., Nature 459, 546 (2009).

[28] T. Yamamoto et al., Phys. Rev. B 82, 184515 (2010).

[29] P. Roushan et al., In preparation (2014).

[30] E. Knill, D. Leibfried, R. Reichle, J. Britton, R. B. Blakestad, J. D. Jost, C. Langer, R. Ozeri, S. Seidelin, and D. J. Wineland, Phys. Rev. A 77, 012307 (2008).

[31] E. Magesan et al., Phys. Rev. Lett. 109, 080505 (2012).

[32] E. Lucero et al., Phys. Rev. Lett. 100, 247001 (2008).

[33] John. M. Martinis and Michael R. Geller, arXiv:1402.5467 (2014). 


\title{
Supplementary information for "Qubit architecture with high coherence and fast tunable coupling"
}

\author{
Yu Chen ${ }^{1},{ }^{*}$ C. Neill ${ }^{1},{ }^{*}$ P. Roushan ${ }^{1},{ }^{*}$ N. Leung ${ }^{1}$, M.l Fang ${ }^{1}$, R. Barends ${ }^{1}$, B. Campbell ${ }^{1}$, Z. Chen ${ }^{1}$, B. Chiaro ${ }^{1}$, A. \\ Dunsworth $^{1}$, E. Jeffrey ${ }^{1}$, J. Kelly ${ }^{1}$, A. Megrant ${ }^{1}$, J. Y. Mutus ${ }^{1}$, P. J. J. O’Malley ${ }^{1}$, C. M. Quintana ${ }^{1}$, D. Sank ${ }^{1}$, \\ A. Vainsencher ${ }^{1}$, J. Wenner ${ }^{1}$, T. C. White ${ }^{1}$, Michael R. Geller ${ }^{2}$, A. N. Cleland ${ }^{1}$, and John M. Martinis ${ }^{1 \dagger}$ \\ ${ }^{1}$ Department of Physics, University of California, \\ Santa Barbara, California 93106-9530, USA and \\ ${ }^{2}$ Department of Physics and Astronomy, University of Georgia, Athens, Georgia 30602, USA
}

\section{CALIBRATION}

A key aspect of our design is the independent control of the qubit frequency and inter-qubit coupling. The resonance frequency of the individual qubits depends on the impedance of the coupling circuit; this is true for any coupling scheme. In our design, the total qubit inductance $L$ is given by

$$
\begin{aligned}
L & =L_{J}+L_{g} \|\left(L_{g}+L_{c}\right) \\
& =L_{J}+L_{g}-M
\end{aligned}
$$

where || stands for "in parallel with" and $\mathrm{M}$ is the mutual inductance given in Eq. (1) of the main text. Changing the inter-qubit coupling is achieved by changing the mutual inductance, which additionally shifts the qubit's resonance frequency. We are able to compensate for this change in inductance using the tunable inductance of the qubit junction $L_{J}$. The compensation is achieved by first measuring the qubit frequency $\omega$ as a function of the qubit flux bias $\Phi_{Q}$ and then as a function of coupler bias $\Phi_{C}$. The qubit frequency is given by $\omega=1 / \sqrt{L C}-\alpha$ where $\mathrm{C}$ is the qubit capacitance and $\alpha$ is the anharmonicity. Solving this expression for $\mathrm{L}$ and using the measured data for $\omega$ yields $L\left(\Phi_{C}\right)$ and $L\left(\Phi_{Q}\right)$. From the first expression we determine the change in inductance $\Delta L$ due to a change in $\Phi_{C}$. Using the second expression we calculate the qubit flux bias required to shift $L$ by $-\Delta L$. Summing these two terms yields zero net change in the qubit inductance. Note that the number of measurements required to compensate for the frequency shift scales linearly with the number of qubits and couplers.

The results of this compensation protocol are shown in Fig. 1(a). For each value of the coupler flux bias, we sweep the microwave drive frequency and measure the excited state probability $P_{1}$. The frequency is almost completely independent of the coupler bias, with a standard deviation of $110 \mathrm{kHz}$. We fit each vertical column of data for a peak and plot the results in blue in Fig. 1(b). We perform an identical measurement without calibration and overlay the results in green. We see that the qubit frequency shifts by over $60 \mathrm{MHz}(\sim g / 2 \pi)$ as we vary the coupler bias.

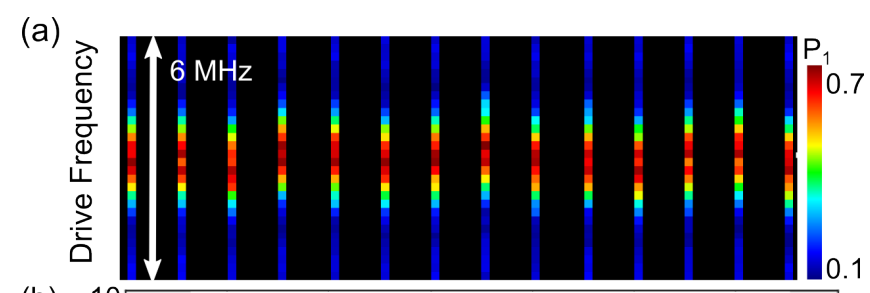

(b)

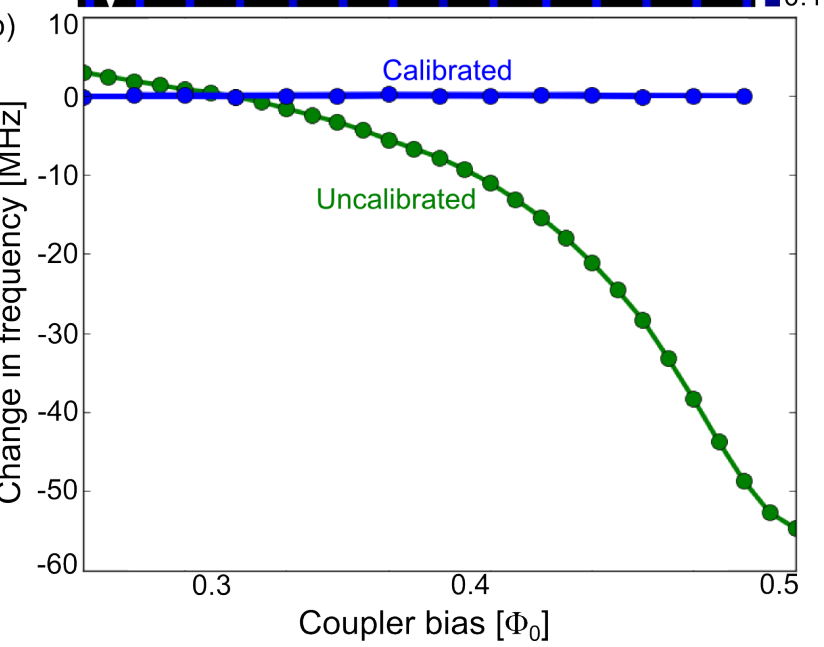

FIG. 1: (a) The frequency of $Q_{1}$, as a function of the coupler flux bias while the second qubit is far detuned. For each value of the coupling strength, we compensate the frequency shift due to the change in inductance, sweep the microwave drive frequency and measure the qubit excited state probability $P_{1}$. Each line is fit for a peak, with the results plotted in panel (b) in blue. The associated standard deviation is $110 \mathrm{kHz}$. The same experiment is performed without the calibration and overlayed in green.

\section{COHERENCE}

The most important part of constructing this tunable coupling architecture is to maintain the coherence inherent in the Xmon design. There are two primary sources of loss associated with the modifications that we have made: capacitive coupling to surface defects on the coupling structure and inductive coupling to the added bias line. The voltage divider created by $L_{J}$ and $L_{g}$ reduce capacitive losses by a factor of over 2000 . The coupler bias line has a mutual inductance to the junction loop of $1 \mathrm{pH}$; this $1 \mathrm{pH}$ coupling to a $50 \mathrm{Ohm}$ line introduces a decoher- 

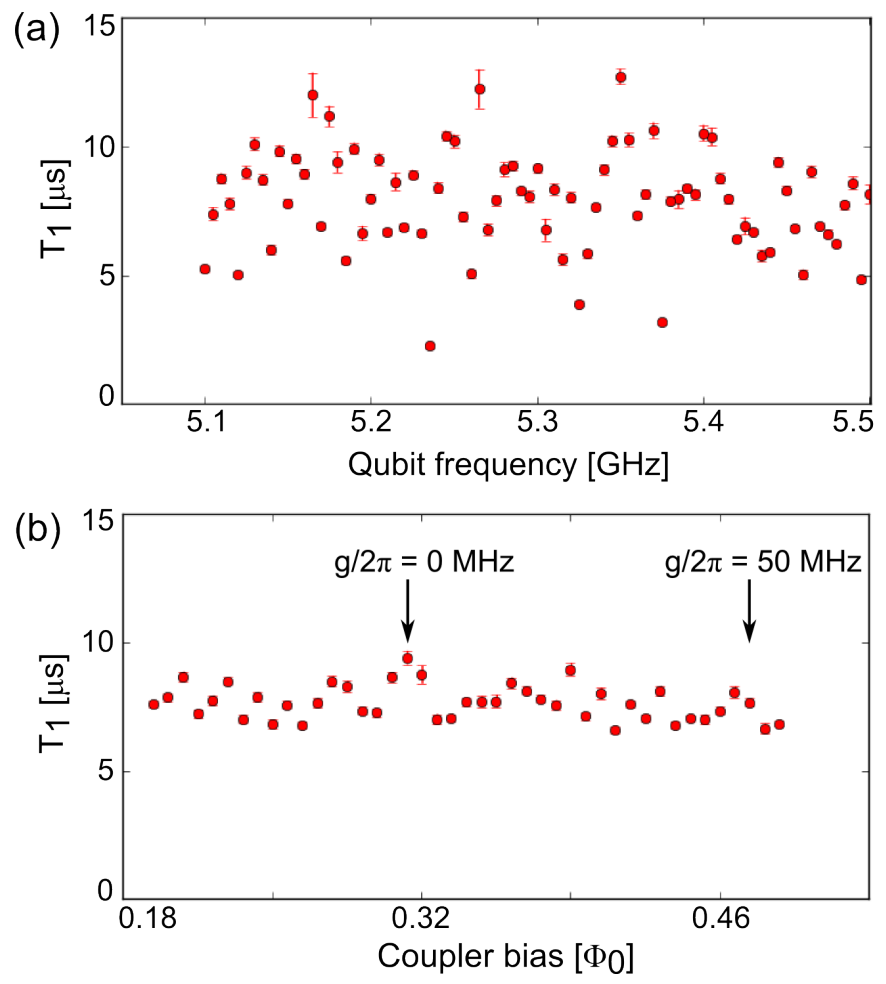

FIG. 2: (a) $T_{1}$ of $Q_{1}$ as a function of the qubit frequency, when $g=0$. These results are comparable to that of the Xmon with similar capacitor geometry and growth conditions. (b) $T_{1}$ of $Q_{1}$ as a function of the coupler bias, when the qubit frequency is set to $5.3 \mathrm{GHz}$. We find no dependence of the $T_{1}$ on the coupling strength.

ence source with an associated $T_{1}$ of greater than $200 \mu \mathrm{s}$ at $80 \mathrm{MHz}$ of coupling. We measure $T_{1}$ as a function of the qubit frequency and plot the results in Fig. 2(a). These rsults are comparable to the performance of previous Xmon devices with similar capacitor geometry and growth conditions. We observe no indication that the $T_{1}$ is reduced as we vary the coupling strength, with data shown in Fig. 2(b).

\section{ZERO COUPLING}

An important application of tunable coupling is to isolate individual qubits for local operations by turning off the coupling. We characterize the zero coupling of our architecture using a modified swap spectroscopy measurement. We bring the two qubits on resonance and vary the coupler flux bias. For each value of the coupling strength, we excite $Q_{1}$, wait a variable delay time and measure its excited state probability. As the results in Fig. 3(a) show, over a wide range of biases, the two qubits can interact and swap an excitation. At a coupler bias of $\sim 0.32 \Phi_{0}$, there is no excitation swapping between the two qubits, indicating that the coupling is turned off. Focusing on zero coupling, we examine the excited state probability

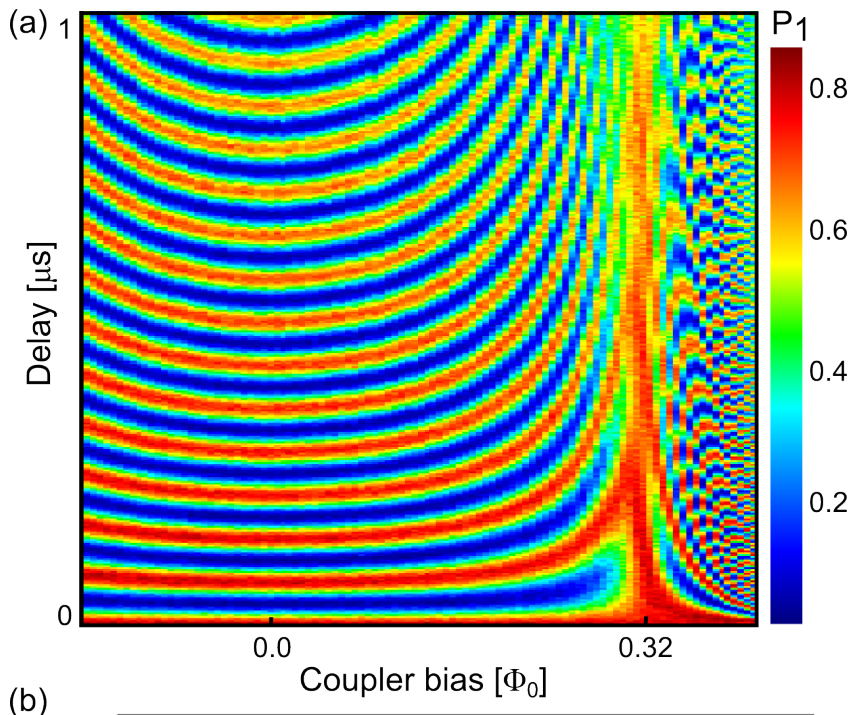

(b)

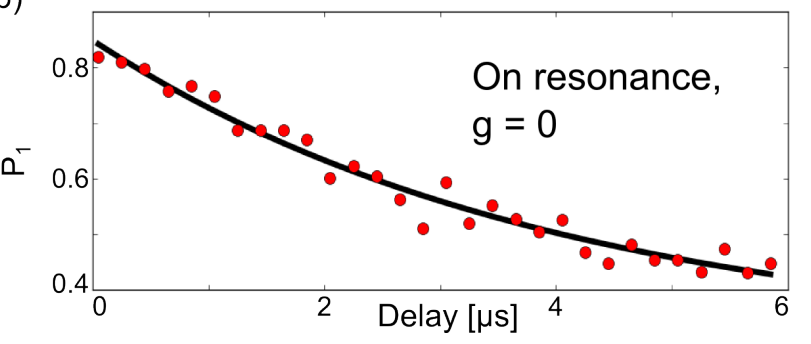

FIG. 3: (a) Swap spectroscopy for $Q_{1}$, as a function of the coupler flux bias, with the two qubits on resonance. For each value of the coupling strength, we excite $Q_{1}$, wait a variable delay time and measure the excited state probability $P_{1}$. We see no excitation swapping between the two qubits when coupler bias is $\sim 0.32 \Phi_{0}$, indicating that the coupling is turned off. (b) We set the coupler bias to this value and examine the excited state probability $P_{1}$ of $Q_{1}$ over an extended delay time. We see no indication of swapping between the two qubits after $6 \mu$ s (placing an upper bound on residual coupling of $50 \mathrm{kHz}$.)

$P_{1}$ of $Q_{1}$ over a extended delay time, with the results shown in Fig. 3(b). We see no indication of swapping between the two qubits after $6 \mu \mathrm{s}$. This places an upper bound on residual coupling of $50 \mathrm{kHz}$, resulting in an on $/$ off ratio $>1000$.

\section{CZ ERROR BUDGET}

We perform two measurements to determine the sources of errors in our $\mathrm{CZ}$ gate. The dominant contribution to the $0.93 \%$ error comes from decoherence. We measure this contribution by performing interleaved randomized benchmarking on a $20 \mathrm{~ns}$ two-qubit idle gate, with $g=0$. We first measure a reference curve without the interleaved idle and plot the data in red in Fig.4(a). We then perform an interleaved randomized benchmarking sequence in which we insert an idle gate between each random Pauli gate, and overlay the data in blue. Com- 
(a)
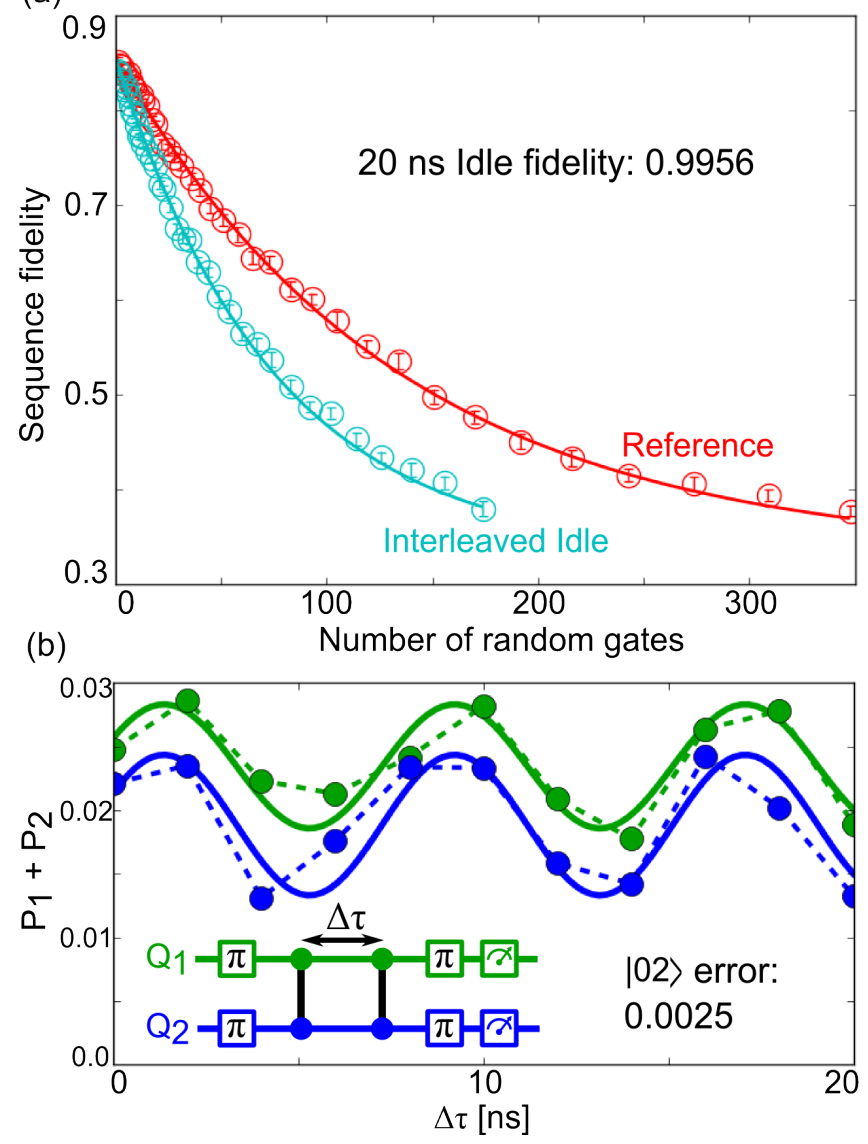

FIG. 4: (a) Interleaved randomized benchmarking on a $20 \mathrm{~ns}$ two-qubit idle gate $(g=0)$. We extract a fidelity of $99.56 \%$, which suggests a decoherence error of $0.66 \%$ for the $30 \mathrm{~ns} \mathrm{CZ}$ gate. (b) Inset: The pulse sequence for the Ramsey error filter technique. Main panel: The measured excited state probability $P_{1}+P_{2}$ as a function of the delay between two $\mathrm{CZ}$ gates. We observe the expected sinusoidal oscillation with a peak-to-peak amplitude of $1 \%$. The non-adiabatic error from $|02\rangle$ state leakage is $1 / 4$ of the oscillation amplitude and is therefore $\sim 0.25 \%$.

paring these two curves allows us to extract a fidelity of $99.56 \%$ for a $20 \mathrm{~ns}$ two-qubit idle gate. Scaling this error rate by a factor of 1.5 to account for the relative length of the $\mathrm{CZ}$ yields an error from decoherence of $\sim 0.66 \%$.

The next largest contribution to errors are from nonadiabatic transitions from the $|11\rangle$ to $|02\rangle$ state. We directly measure this transition using a Ramsey error filter technique [1]; the pulse sequence is shown inset in Fig. 4(b). We initialize the system in the $|11\rangle$ state and then apply two $\mathrm{CZ}$ gates separated by a variable delay time. Afer applying a $\pi$-pulse to each qubit, we measure the uncorrelated excited state probability for each qubit. The results are shown in Fig.4(b), where we see the expected oscillations that result from the interference between two $\mathrm{CZ}$ gates. The frequency of the oscillation is set by the detuning of the $|11\rangle$ and $|02\rangle$ states which was $130 \mathrm{MHz}$, corresponding to a period of $8 \mathrm{~ns}$. The $|02\rangle$ state leakage error is given as $1 / 4$ of the oscillation amplitude (peak-to-peak). For our $30 \mathrm{~ns} \mathrm{CZ}$ gate, we measured a non-adiabatic error of $\sim 0.25 \%$. This is suprisingly small considering such a short gate time, and can be exponentially surpressed with increasing gate length.

\section{TRANSMON PHYSICS}

The operation of the transmon has been previously described in detail [2]. Here, we give a simplified calculation in the phase basis that is useful to describe more complex transmon circuits, as for the gmon architecture.

Since the transmon produces qubit behavior from a weak non-linearity, we first review the physics of a linear inductor-capacitor $(L C)$ oscillator. In terms of physical variables charge $q$ and flux $\Phi$, the oscillator Hamiltonian is given by

$$
\hat{H}_{o}=\frac{\hat{q}^{2}}{2 C}+\frac{\hat{\Phi}^{2}}{2 L} .
$$

Here the quantum operators of flux and charge obey the standard commutation relation $[\hat{\Phi}, \hat{q}]=i \hbar$. The oscillator frequency is the classical value $\omega=1 / \sqrt{L C}$, and eigenstates $m$ have energy $E_{m}=\hbar \omega(m+1 / 2)$. The ground state wavefunction is given by

$$
\Psi_{0}(\Phi) \propto \exp \left[-(\omega C / 2 \hbar) \Phi^{2}\right]
$$

Note that the width of the wavefunction is set by the oscillator impedance $Z_{o}=1 / \omega C=\omega L=\sqrt{L / C}$. Varying this impedance changes the widths of the charge and flux wavefunctions, as illustrated in TableI. The impedance is also important since it is used to describe how strongly the oscillator couples to other modes. The flux and charge operators are conveniently expressed in terms of the raising and lowering operators

$$
\begin{aligned}
& \hat{\Phi}=(\hbar / 2 \omega C)^{1 / 2}\left(a^{\dagger}+a\right) \\
& \hat{q}=(\hbar \omega C / 2)^{1 / 2} i\left(a^{\dagger}-a\right) .
\end{aligned}
$$

For a tunnel junction with shunting capacitor, the charge on the metal island takes on discrete values corresponding to the number of Cooper pairs $n$. The Hamiltonian for this system is given by

$$
\hat{H}_{t}=4 E_{c}\left(\hat{n}-n_{g}\right)^{2}-E_{J} \cos \hat{\delta},
$$

TABLE I: Table of relative width of charge and flux wavefunctions as capacitance $C$ (and impedance $Z_{o}$ ) are changed.

\begin{tabular}{|c|c|c|c|}
\hline$C$ & $Z_{o}$ & $\left\langle\hat{q}^{2}\right\rangle$ & $\left\langle\hat{\Phi}^{2}\right\rangle$ \\
\hline small & large & small & large \\
large & small & large & small \\
\hline
\end{tabular}


where $E_{c}=e^{2} / 2 C$ is the charging energy and $E_{J}=$ $I_{0} \Phi_{0} / 2 \pi$ is the Josephson energy from the tunnel junction, with critical current $I_{0}$. The normalized coordinates are related to ordinary electrical variables by $\hat{q}=2 e \hat{n}$ and $\hat{\Phi}=\left(\Phi_{0} / 2 \pi\right) \hat{\delta}$, and thus their commutation relation is $[\hat{\delta}, \hat{n}]=i$. Here we have included a continuous charge bias $n_{g}$, produced for example by a small coupling capacitor with voltage bias. The Josephson term can be written as $\cos \hat{\delta}=[\exp (+i \hat{\delta})+\exp (-i \hat{\delta})] / 2$, corresponding to number displacement operators $\exp ( \pm i \hat{\delta})$ that couple states that differ by one in the number of Cooper pairs.

The form of the solution for this Hamiltonian depends on the ratio of these two energies. For small capacitance where $E_{c} \gg E_{J}$, the "Cooper-pair box" limit, the charging energy dominates, and the eigenstates are described by one or the superposition of two number states. The states sensitively depend on the gate charge $n_{g}$. This is death to qubit physics, since fluctuations of gate charge from the movement of trapped charge around the junction produces large qubit decoherence from dephasing.

We are interested in the large capacitance "transmon" limit, where $E_{J} \gg E_{c}$. Here, the dependence of qubit energy on the gate charge becomes exponentially small, so qubit decoherence from charge fluctuations essentially vanishes. To understand this, note that for large capacitance the phase fluctuations are small. The potential $\cos \hat{\delta}$ can then be expanded in powers of $\hat{\delta}$, with the lowest non-trivial term giving an inductive energy. First considering the case $n_{g}=0$, one obtains a harmonic oscillator-like Hamiltonian

$$
H_{t o}=4 E_{c} \hat{n}^{2}+\left(\Phi_{0} / 2 \pi\right)^{2} \hat{\delta}^{2} / 2 L_{J},
$$

where the Josephson inductance is $L_{J}=\left(\Phi_{0} / 2 \pi\right)^{2} / E_{J}=$ $\Phi_{0} / 2 \pi I_{0}$. We can thus use harmonic oscillator solutions as the basis eigenstates for perturbation theory.

Note that formally the charge wavefunction is a deltafunction comb with spacings $2 e$ in charge, with amplitudes given by the harmonic oscillator solution. The charge comb corresponds to a phase wavefunction periodic in $2 \pi$. As the capacitance increases, the number of states in the charge wavefunction increases, so that the relative separation of the teeth in the charge comb become so closely spaced as to look like the normal continuous solution for the harmonic oscillator. In phase, this implies the wavefunction is so localized in phase that the $2 \pi$ periodicity does not matter.

The phase wavefunction has a width $\left\langle\hat{\delta}^{2}\right\rangle$ that can be computed using the exponential term in the wavefunction given by Eq. (3)

$$
1=\frac{\omega C}{\hbar}\left(\frac{\Phi_{0}}{2 \pi}\right)^{2}\left\langle\hat{\delta}^{2}\right\rangle
$$

which gives

$$
\begin{aligned}
\left\langle\hat{\delta}^{2}\right\rangle & =\sqrt{8 E_{c} / E_{J}} \\
& =Z_{J} /\left(R_{K} / 8 \pi\right),
\end{aligned}
$$

where in the last equation $R_{K}=h / e^{2}=25.8 \mathrm{k} \Omega$ is the resistance quantum, and $R_{K} / 8 \pi=1.026 \mathrm{k} \Omega$. The phase basis works well when the mean quantum fluctuation of the phase is small, which corresponds to a small $E_{c} / E_{J}$ ratio or a junction impedance $Z_{J}=\sqrt{L_{J} / C}$ much less than $1 \mathrm{k} \Omega$.

The effect of the gate charge $n_{g}$ in the Hamiltonian can be computed by noting that this offset in the operator $\hat{n}$ can be accounted for by the displacement operator $\exp \left(i n_{g} \hat{\delta}\right)$ applied to the solution of $H_{t}$ with $n_{g}=0$. This is equivalent to imposing periodic boundary conditions at the phase $\delta= \pm \pi$

$$
\Psi(-\pi)=\Psi(\pi) e^{i 2 \pi n_{g}} .
$$

We can estimate the effect of this boundary condition on the eigenstates by noting that it should be proportional to the probability of the wavefunction at $\delta=\pi$. Using the harmonic oscillator solution, the magnitude of the modulation of eigenstate energy from charge $n_{g}$ should scale approximately as

$$
\begin{aligned}
\Delta E & \propto\left|\Psi_{0}(\delta=\pi)\right|^{2} \\
& =\exp \left[-(\omega C / \hbar)\left(\Phi_{0} / 2\right)^{2}\right] \\
& =\exp \left[-\left(\pi^{2} / 8\right) \sqrt{8 E_{J} / E_{c}}\right] .
\end{aligned}
$$

We may calculate the exponential factor precisely by including the non-linear junction energy. Using the WKB theory, with constants $2 m=1 / 4 E_{c}$ and $\hbar=1$ from Eq. (6) and its commutation relation, we find

$$
\begin{aligned}
\left|\Psi_{0}(\pi)\right|^{2} & =\exp \left[-2 \int_{0}^{\pi} d \delta \sqrt{\left(1 / 4 E_{c}\right) E_{J}(1-\cos \delta)}\right] \\
& =\exp \left[-\sqrt{8 E_{J} / E_{c}}\right]
\end{aligned}
$$

matching the result of Ref. [2]. A large $E_{J} / E_{c}$ ratio gives exponentially low sensitivity to charge noise.

Note that the phase qubit has vanishing sensitivity to charge noise for two reasons. First, the ratio of $E_{J} / E_{c}$ is even larger than for the transmon. Second, the latest versions of the device used a shunting inductor for current biasing. The continuous flow of charge across the junction then shunts any DC change in charge bias. This latter effect is the purpose of the inductor shunt in the fluxonium device.

For completeness, we compute the change in the harmonic oscillator energy eigenvalues due to the cosine nonlinearity. Starting from

$$
\cos \hat{\delta} \simeq 1-\hat{\delta}^{2} / 2+\hat{\delta}^{4} / 24
$$

the correction to the energy from the fourth order term is

$$
\begin{aligned}
\Delta E_{m} & =-E_{J}\left\langle m\left|\hat{\delta}^{4}\right| m\right\rangle / 24 \\
& =-\frac{E_{J}}{24}\left(\frac{\hbar}{2 \omega C}\right)^{2}\left(\frac{2 \pi}{\Phi_{0}}\right)^{4}\left\langle m\left|\left(a^{\dagger}+a\right)^{4}\right| m\right\rangle
\end{aligned}
$$


The matrix element can be calculated by using the square $\left(a^{\dagger}+a\right)^{2}=a^{\dagger 2}+a^{2}+2 a^{\dagger} a+1$, giving

$$
\begin{gathered}
\left\langle m\left|\left(a^{\dagger}+a\right)^{4}\right| m\right\rangle=\left\langle m\left|a^{\dagger 2} a^{2}+a^{2} a^{\dagger 2}+\left(2 a^{\dagger} a+1\right)^{2}\right| m\right\rangle \\
\quad=m(m-1)+(m+1)(m+2)+(2 m+1)^{2} \\
\quad=6 m^{2}+6 m+3
\end{gathered}
$$

where in the first equation we have only kept terms that leave $|m\rangle$ unchanged. The change in energy between adjacent states is

$$
\Delta\left(E_{m}-E_{m-1}\right)=-m E_{c}
$$

as expected. As the unperturbed oscillator frequency can be written as $\hbar \omega=\sqrt{8 E_{J} E_{c}}$, the fractional change in qubit frequency is $\sqrt{E_{c} / 8 E_{J}}$.

\section{A. Series Inductance}

We next consider how this physics changes when including an inductance $L$ in series with the Josephson junction. The total phase across the two elements is given by $\delta=\delta_{L}+\delta_{J}$. The conservation of current at the node between the two elements gives the constraint $I_{L}=I_{0} \sin \delta_{J}$, which then can be used to relate the individual phase changes and their derivative

$$
\begin{aligned}
\delta_{L} / L & =\sin \delta_{J} / L_{J 0} \\
d \delta_{L} / L & =d \delta_{J} \cos \delta_{J} / L_{J 0},
\end{aligned}
$$

where we have defined $L_{J 0}=\Phi_{0} / 2 \pi I_{0}=\left(\Phi_{0} / 2 \pi\right)^{2} / E_{J}$ as the Josephson inductance at zero current.

The WKB theory gives a charge sensitivity that includes both Josephson and inductor energies

$$
\begin{aligned}
& -\ln \left|\Psi_{0}(\pi)\right|^{2} \\
& =\sqrt{\frac{1}{E_{c}}} \int_{0}^{\pi} d \delta \sqrt{E_{J}\left(1-\cos \delta_{J}\right)+\left(\delta_{L} \Phi_{0} / 2 \pi\right)^{2} / 2 L} \\
& =\sqrt{\frac{E_{J}}{E_{c}}} \int_{0}^{\pi} d \delta_{J}\left[1+\left(L / L_{J 0}\right) \cos \delta_{J}\right] \\
& \quad \times \sqrt{1-\cos \delta_{J}+\left(L / 2 L_{J 0}\right) \sin ^{2} \delta_{J}} \\
& \simeq \sqrt{8 E_{J} / E_{c}}\left(1-0.166 L / L_{J 0}\right),
\end{aligned}
$$

where the integral was evaluated numerically. The linear expansion in Eq. (28) is quite good for $L / L_{J 0} \leq 1$

The nonlinearity in the energy levels can be evaluated by noting that the quantum fluctuations of the phase is small, so that we can use the linear relation for phase change $\delta_{L} / L=\delta_{J} / L_{J 0}$. The junction phase can then be found using an inductance divider relation

$$
\delta_{J}=\frac{L_{J 0}}{L+L_{J 0}} \delta .
$$

Following Eq. (19), the change in energy eigenvalues is proportional to $\left\langle\hat{\delta}_{J}^{4}\right\rangle=\left\langle\hat{\delta}^{4}\right\rangle /\left(1+L / L_{J 0}\right)^{4}$, giving

$$
\begin{aligned}
\Delta\left(E_{m}-E_{m-1}\right) & =-\frac{E_{J}}{24}\left(\frac{\hbar}{2 \omega C}\right)^{2}\left(\frac{2 \pi}{\Phi_{0}}\right)^{4} \frac{12 m}{\left(1+L / L_{J 0}\right)^{4}} \\
& =-m E_{c} \frac{1}{\omega^{2} L_{J 0} C} \frac{1}{\left(1+L / L_{J 0}\right)^{4}} \\
& =-m E_{c} \frac{1}{\left(1+L / L_{J 0}\right)^{3}}
\end{aligned}
$$

where for the last equation we have used the resonance condition $\omega^{2}=1 /\left(L+L_{J 0}\right) C$. We see that the extra linear inductance lowers the nonlinearity coming from the junction.

* These authors contributed equally to this work

$\dagger$ Electronic address: martinis@physics.ucsb.edu

[1] Erik Lucero, M. Hofheinz, M. Ansmann, Radoslaw C. Bialczak, N. Katz, Matthew Neeley, A.D. O'Connell, H. Wang, A. N. Cleland, John M. Martinis Phys. Rev. Lett. 100, 247001 (2008).

[2] Jens Koch, T.M. Yu, J.M. Gambetta, A.A. Houck, D.I Schuster, J. Majer, A. Blais, M.H. Devoret, S.M. Girvin, and R.J. Schoelkopf, Phys. Rev. A 76, 042319 (2007). 